\title{
Atuação do enfermeiro na prevenção de lesão por pressão: uma revisão integrativa da literatura
}

\author{
Nurse's role in the prevention of pressure injury: an integrative literature review
}

Actuación del enfermero en la prevención de lesiones por presión: una revisión integral de la literatura

Douglas Vinicius dos Santos Feitosa ${ }^{1 *}$, Noemia Santos de Oliveira Silva ${ }^{1}$, Fabiana Navajas Moreira Pereira $^{1}$, Thaynara Fontes Almeida ${ }^{1}$, Adriana dos Santos Estevam².

\section{RESUMO}

Objetivos: Revisar artigos que destacam o papel do enfermeiro frente avaliação e a prevenção da lesão por pressão. Métodos: Trata-se de uma pesquisa exploratória de caráter bibliográfico, das bases de dados eletrônicas Scientific Electronic Library Online, Base de Dados de Enfermagem e Latino-americana e do Caribe em Ciências da Saúde. Os critérios de inclusão foram: artigos originais, completos e em português, publicados entre 2014 e 2018, em periódicos reconhecidos pelo QUALIS e que abordassem a auditoria dos cuidados de enfermagem e o gerenciamento em saúde no que tange a lesão por pressão (LP). Resultados: Foram mencionadas ações de prevenção como: avaliação do risco de LP pela escala de Braden, o aporte nutricional balanceado, utilização de filme transparente de poliuretano e a placa hidrocoloide, reposicionamento planejado em angulação e com maior frequência, redução da exposição da pele à umidade e a utilização de artigos que modificam o pH da pele. Considerações finais: Torna-se relevante a equipe multiprofissional, especialmente a equipe de enfermagem, a adoção de medidas voltadas para prevenção e condução deste agravo, sendo essencial associar teoria e prática baseadas em diretrizes com evidências que possam conduzir à prática clínica efetiva, com o intuito de diminuir a incidência de LP.

Palavras-chave: Lesão por pressão, Prevenção, Promoção de saúde, Enfermagem.

\section{ABSTRACT}

Objectives: Review articles that highlight the role of nurse front evaluation and the prevention of pressure injury. Methods: This is an exploratory research of bibliographic character, of the electronic databases Scientific Electronic Library Online, Database of Nursing and Latin American and Caribbean in Health Sciences. The inclusion criteria were: original articles, complete and in Portuguese, published between 2014 e 2018, in journals recognized by QUALIS and that addressed the audit of nursing care and health management in relation to pressure injury (LP). Results: Prevention actions were mentioned as: risk assessment of LP by Braden scale, balanced nutritional contribution, use of transparent polyurethane film and hydrocoloid plate planned repositioning at angulation and more frequently, reducing skin exposure to moisture and using articles that modify skin pH. Final considerations: The multiprofessional team becomes relevant, especially the nursing team, the adoption of measures aimed at preventing and conducting this disease, it is essential to associate theory and practice based on guidelines with evidence that can lead to effective clinical practice, in order to reduce the incidence of LP.

Key words: Pressure Injury, Prevention, Health Promotion, Nursing.

${ }^{1}$ Centro Universitário Estácio de Sergipe, Aracaju - SE. *E-mail: viniciusdouglas484@gmail.com

2 Universidade Federal de Sergipe, Aracaju - SE. 


\section{RESUMEN}

Objetivos: Revisar artículos que destacan el papel del enfermero frente evaluación y la prevención de la lesión por presión. Métodos: Se trata de una investigación exploratoria de carácter bibliográfico, de las bases de datos electrónicas Scientific Electronic Library Online, Base de Datos de Enfermería y Latinoamericana y del Caribe en Ciencias de la Salud. Los criterios de inclusión fueron: artículos originales, completos y en portugués, publicados entre 2014 y 2018, en revistas reconocidas por QUALIS y que abordasen la auditoría de los cuidados de enfermería y la gestión en salud en lo que se refiere a la lesión por presión (LP). Resultados: Se mencionaron acciones de prevención como: evaluación del riesgo de LP por la escala de Braden, el aporte nutricional equilibrado, uso de película transparente de poliuretano y la placa hidrocoloide reposicionamiento planificado en angulación y con mayor frecuencia, reducción de la exposición de la piel a la humedad y el uso de artículos que modifican el pH de la piel. Consideraciones finales: Se hace relevante el equipo multiprofesional, especialmente el equipo de enfermería, la adopción de medidas dirigidas a la prevención y conducción de este agravio, es esencial asociar teoría y práctica basadas en directrices con evidencias que puedan conducir a la práctica clínica efectiva, con el fin de disminuir la incidencia de LP.

Palabras clave: Lesión por presión, Prevención, Promoción de salud, Enfermería.

\section{INTRODUÇÃO}

A lesão por pressão (LP) é definida como uma região delimitada pela morte celular desenvolvida quando uma determinada área do corpo sofre pressão entre uma proeminência óssea e uma superfície dura por longo período (SILVA CPC, et al., 2016). Dessa forma, pacientes que apresentam imobilidade física no leito, modificações sensoriais, alterações na circulação periférica, nutrição deficitária, imunodeprimidos ou idosos tem um maior risco para desenvolvimento de LP (MORO JV e CALIRI MHL, 2016). Nessa perspectiva, a lesão por pressão tem se tornado um enorme problema de saúde pública, principalmente em pacientes crônicos que se encontram na terceira idade internados ou em tratamento a nível domiciliar, dificultando o tratamento, sendo prolongado e dispensivo, o que corrobora para a premissa da prevenção (DEBON R, et al., 2018).

De acordo com os dados da National Pressure Ulcer Advisory Panel, os EUA, a prevalência de lesão por pressão em instituições hospitalares é de $15 \%$ e a incidência é de $7 \%$. No Reino Unido, novos casos de LP acometem entre $4 \%$ a $10 \%$ dos pacientes internados nos hospitais. No Brasil, embora existam poucas pesquisas sobre incidência e prevalência de LP, um estudo realizado em um hospital geral universitário mostrou uma incidência de 39,81\% (NPUAP, 2014).

Silva SPC, et al. (2016), afirmam que a incidência da LP é considerada um importante indicador da qualidade assistencial de enfermagem que permite pesquisar os casos de acordo com sua distribuição, pacientes que se encontram em risco e região anatômica o local em que ocorre com maior frequência. Diante disso, a enfermagem apresenta-se como uma ciência que tem como característica o cuidado humanizado. Neste sentido, o aspecto preventivo, bem como o de promoção da saúde, busca nortear a prática assistencial para minimizar os índices de lesão por pressão (SOARES CF e HEIDEMANN ITSB, 2018).

Para Nogueira PC, et al. (2015) e Aroldi JBC, et al., (2018) as LP podem ser prevenidas com a adoção de práticas assistenciais, educação dirigida tanto para equipe de enfermagem quanto para o cuidador familiar, com estratégias que visem à continuação do cuidado a nível domiciliar, corroborando a indispensabilidade de aprendizagem e aplicação prática mais assertiva por parte dos enfermeiros.

Sob tal perspectiva, o enfermeiro, na qualidade de profissional de saúde, comprometido na assistência ao paciente hospitalizado, deve planejar medidas de cuidados adequados capazes de promover a saúde, a adesão ao tratamento e estimular a equipe de enfermagem a prestar assistência humanizada (MORO JV e CALIRI MHL, 2016). Desta forma, o presente estudo apresenta como contribuição a formulação de diretrizes clínicas e políticas públicas para nortear as práticas preventivas e ações de enfermagem no gerenciamento assistencial do cuidado, a fim de reduzir este agravo (RIOS BL, et al., 2016). 
Esse estudo justifica-se devido à dificuldade apresentada pelos profissionais de enfermagem na prevenção e avaliação das lesões por pressão, uma vez que, uma avaliação adequada da pele proporciona sua prevenção e a implementação de medidas terapêuticas eficaz para cicatrização tecidual. Dessa forma, a pesquisa objetivou analisar estudos que ressaltam a atuação do enfermeiro na avaliação e prevenção de lesão por pressão, tendo em vista que a assistência de enfermagem à pacientes portadores de feridas é uma especialidade da enfermagem que requer conhecimento específico.

\section{MÉTODOS}

Estudo de revisão integrativa da literatura com a finalidade de agrupar e resumir o conhecimento científico já produzido sobre o tema investigado, permitindo buscar, avaliar e sintetizar as evidências disponíveis para colaborar com incremento do saber a respeito da temática. A questão que guiou a pesquisa foi: $O$ que há produzido na literatura sobre a atuação do enfermeiro frente à prevenção e avaliação de lesão por pressão?

As buscas das publicações deram-se entre novembro e dezembro de 2018, nas seguintes bases de dados: Literatura Latino-americana e do Caribe em Ciências da Saúde (LILACS), Biblioteca Virtual de Saúde (BVS) e Biblioteca Eletrônica Scientific Electronic Library Online (SciELO), sendo que os artigos encontrados passaram pelo filtro da classificação de periódicos, o QUALIS. Entende-se que estas bases de dados atingem a literatura publicada nos países da América Latina Caribe, referências técnico-científicas brasileiras em enfermagem e periódicos conceituados da área da saúde. Foram utilizados como descritores: Lesão Por Pressão; Prevenção, Promoção da Saúde e Enfermagem.

Os critérios de inclusão foram: artigos originais, completos, no idioma português, publicados entre 2014 e 2018, em periódicos reconhecidos pelo Coordenação de Aperfeiçoamento de Pessoal de Nível Superior CAPES com QUALIS e que abordassem a auditoria dos cuidados de enfermagem e o gerenciamento em saúde. Salienta- se que a busca foi realizada de forma ordenada, BVS, LILACS e SciELO, desta maneira as publicações que se encontravam indexadas em mais de uma plataforma, foram selecionadas na primeira busca.

Após a avaliação dos resumos, as produções que atenderam aos critérios descritos anteriormente, foram selecionadas e lidas na íntegra. A busca nas bases de dados resultou em 31 artigos. A seleção inicial considerando os critérios de inclusão e exclusão definiu apenas 21 para análise, especialmente pelo objetivo do presente estudo, os demais artigos foram excluídos por não ser publicados entre o ano de 2014 a 2018. A partir destes artigos, surgiram as seguintes temáticas: medidas preventivas relacionadas às boas práticas assistenciais e instrumentos utilizados na avaliação da pele.

Vale ressaltar que, em relação à análise de dados, o princípio básico que a norteia visa debater os principais resultados evidenciados nas pesquisas convencionais que fazem parte da amostra. Além disso, tem como ponto de partida revisá-los, avaliar os pontos críticos dos estudos incluídos, e, por conseguinte, realizar a comparação com o conhecimento teórico. Assim, convém pontuar que os resultados foram categorizados e inseridos em quadros ou gráficos.

Para coleta de dados foi elaborado um quadro sintético composto pelos seguintes itens: título do artigo, ano, autor, objetivo e metodologia do estudo. A análise dos dados foi executada de forma descritiva, por meio da categorização e síntese das temáticas. Com o intuito de descrever e classificar os resultados, evidenciando o conhecimento produzido sobre o tema proposto, realizou-se a análise, categorização e síntese das temáticas (MENDES KDD, et al., 2008).

\section{RESULTADOS}

A partir dos critérios de busca pré-estabelecidos, nas bases de dados consultadas, foi possível obter referências considerando os critérios de inclusão e de acordo com o objetivo da pesquisa. Sendo dez (48\%) indexadas na base de dados LILACS, três (14\%) indexadas na base de dados SciELO e oito (19\%) na base de dados BVS (Figura 1). 
Figura 1 - Relação de porcentagem dos artigos encontrados na literatura. Aracaju - SE, 2019.

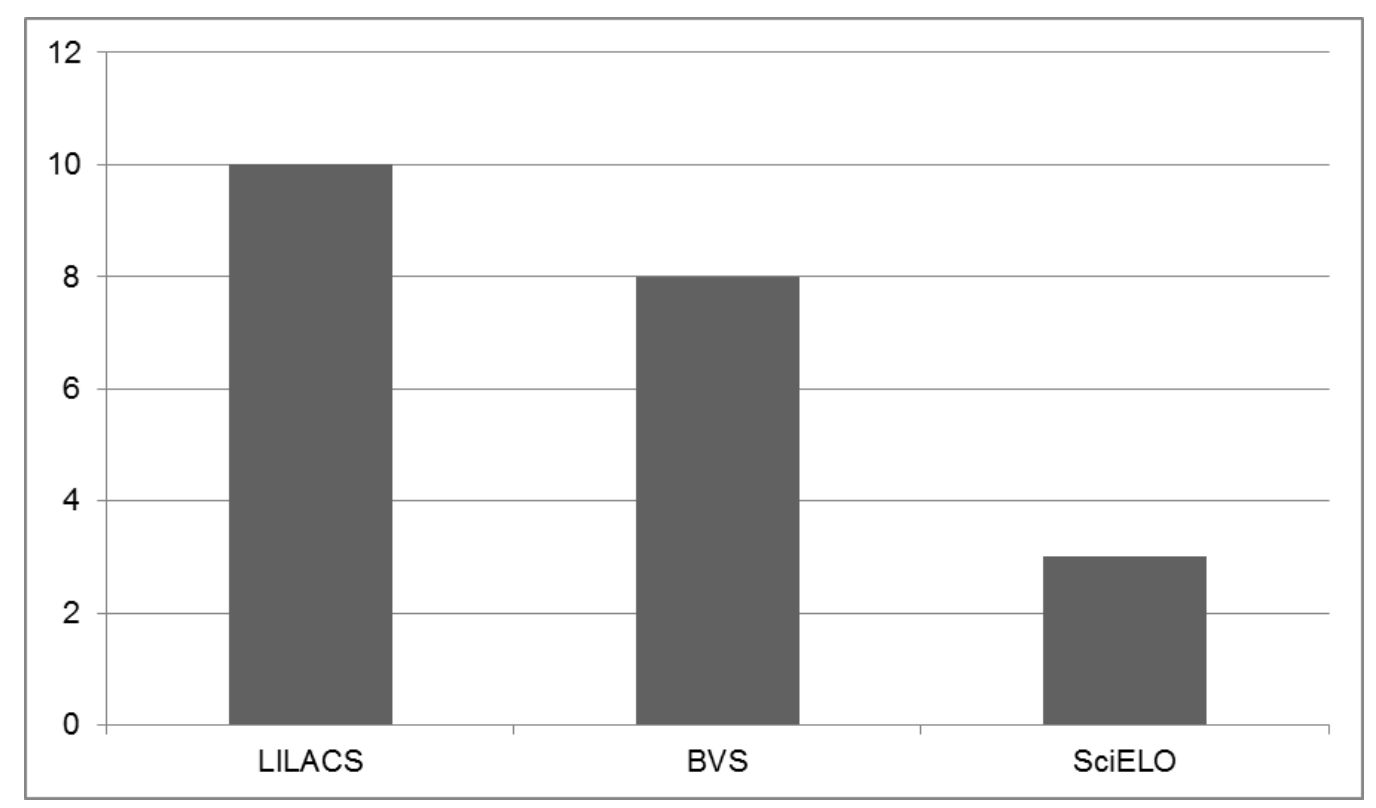

Fonte: Feitosa DVS, et al., 2019.

Como critérios de inclusão foram selecionados artigos publicados entre os anos de 2014 e 2018, a proporção dos estudos publicados por ano foram: 2014 , dois $(9,52 \%), 2015$, cinco $(23,81 \%)$, 2016, seis (28,57\%), 2017, cinco (23,81\%), 2018, três (14,29\%) artigos, sendo estes específicos em quantidade por ano (Figura 2).

Figura 2 - Relação de ano das publicações dos artigos encontrados na literatura. Aracaju - SE, 2019.

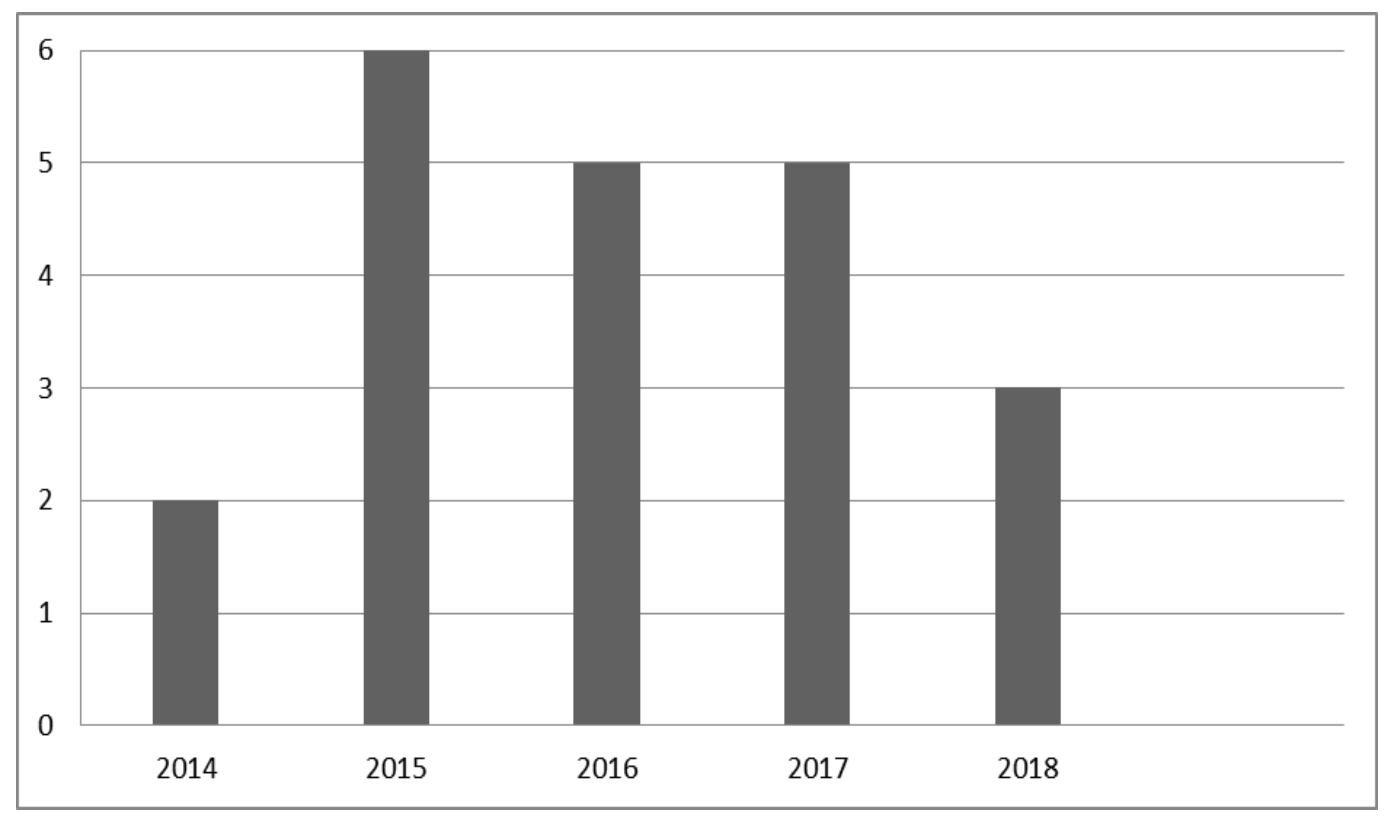

Fonte: Feitosa DVS, et al., 2019.

Para uma melhor compreensão dos artigos selecionados, o Quadro 1 tem como objetivo apresentar as principais características dos 21 artigos incluídos na análise para a efetivação deste estudo. Assim, estão apresentados em ordem crescente com relação ao ano de publicação, título, autores, objetivos e metodologia. 
Quadro 1 - Síntese panorâmica dos artigos selecionados, Aracaju - SE, 2019.

\begin{tabular}{|c|c|c|c|}
\hline Numeração & Título & Objetivo & Métodos \\
\hline $\begin{array}{c}\text { 1-Mauricio } A B \text {, et } \\
\text { al.(2014) }\end{array}$ & $\begin{array}{l}\text { Conhecimentos } r \text { dos } \\
\text { profissionais de enfermagem } \\
\text { sobre úlceras por Pressão na } \\
\text { Clínica Médica. }\end{array}$ & $\begin{array}{l}\text { Identificar os conhecimentos que os profissionais } \\
\text { de enfermagem possuem relacionados às } \\
\text { medidas de prevenção, monitoramento e } \\
\text { tratamento das úlceras por pressão na Clínica } \\
\text { Médica. }\end{array}$ & $\begin{array}{l}\text { Estudo descritivo de abordagem quantitativa realizado } \\
\text { com profissionais de enfermagem em um Hospital } \\
\text { Escola de Curitiba. }\end{array}$ \\
\hline $\begin{array}{l}\text { 2-Barbosa TP, et } \\
\text { al. (2014) }\end{array}$ & $\begin{array}{l}\text { Avaliação do risco de úlcera por } \\
\text { pressão em UTI e assistência } \\
\text { preventiva de enfermagem. }\end{array}$ & $\begin{array}{l}\text { Identificar os pacientes com risco de desenvolver } \\
\text { úlcera por pressão (UPP) em unidade de terapia } \\
\text { (UTI) por meio da escala de Braden e relacionar } \\
\text { esses escores com a assistência de enfermagem } \\
\text { na sua prevenção. }\end{array}$ & $\begin{array}{l}\text { Estudo transversal, prospectivo realizado num hospital } \\
\text { de ensino de porte especial, com } 780 \text { leitos, situado no } \\
\text { interior do estado de São Paulo, em três UTIs: geral, } \\
\text { convênio e emergência denominados por UTI A, B e C, } \\
\text { respectivamente. }\end{array}$ \\
\hline $\begin{array}{l}\text { 3-Nogueira PC, et } \\
\text { al. (2015) }\end{array}$ & $\begin{array}{l}\text { Conhecimento dos cuidadores } \\
\text { de indivíduos com lesão medular } \\
\text { sobre prevenção e de úlcera por } \\
\text { pressão. }\end{array}$ & $\begin{array}{l}\text { Identificar as características sociodemográficas } \\
\text { das pessoas com lesão medular espinhal (LME) } \\
\text { e de seus cuidadores familiares e avaliar o } \\
\text { conhecimento dos cuidadores sobre prevenção } \\
\text { de lesão por pressão. }\end{array}$ & $\begin{array}{l}\text { Estudo descritivo-exploratório, quantitativo, realizado } \\
\text { em um hospital universitário localizado em um interior } \\
\text { dom estado de São Paulo. Participaram } 47 \text { pessoas } \\
\text { com LME e } 47 \text { cuidadores que responderam a um teste } \\
\text { de conhecimento sobre prevenção de LP. }\end{array}$ \\
\hline $\begin{array}{l}\text { 4-Inoue KC e } \\
\text { Matsuda LM } \\
\quad(2015)\end{array}$ & $\begin{array}{l}\text { Avaliação de custo-efetividade } \\
\text { de dois tipos de curativos para } \\
\text { prevenção de úlcera por } \\
\text { pressão. }\end{array}$ & $\begin{array}{l}\text { Analisar a relação custo-efetividade de dois tipos } \\
\text { de curativos para a prevenção de úlcera por } \\
\text { pressão na região sacral. }\end{array}$ & $\begin{array}{l}\text { Pesquisa de analise secundária, comparativa, que } \\
\text { incluiu } 25 \text { participantes dos quais dez utilizaram a } \\
\text { cobertura hidrocoloide e } 15 \text { o filme transparente, para } \\
\text { prevenção de úlcera por pressão na região sacral. }\end{array}$ \\
\hline $\begin{array}{l}\text { 5-Laurenti TC, et } \\
\text { al. (2015) }\end{array}$ & $\begin{array}{l}\text { Gestão Informatizada de } \\
\text { Indicadores de Úlcera Por } \\
\text { Pressão }\end{array}$ & $\begin{array}{l}\text { O objetivo desta pesquisa foi criar um banco de } \\
\text { dados informatizado com indicadores de } \\
\text { gerenciamento de risco para úlceras por pressão } \\
\text { com a finalidade de promover estratégias de } \\
\text { prevenção, controle e tratamento. }\end{array}$ & $\begin{array}{l}\text { Pesquisa descritiva e quantitativa referente à geração } \\
\text { de conhecimentos para elaboração de estratégias que } \\
\text { reduzam a incidência de úlceras por pressão, utilizando } \\
\text { intervenções eficazes. }\end{array}$ \\
\hline $\begin{array}{c}\text { 6-Rocha LES, et } \\
\text { al.(2015) }\end{array}$ & $\begin{array}{l}\text { Prevenção de úlceras por } \\
\text { pressão: avaliação do } \\
\text { conhecimento dos profissionais } \\
\text { de enfermagem. }\end{array}$ & $\begin{array}{l}\text { Avaliar o conhecimento da equipe de } \\
\text { enfermagem sobre medidas de prevenção de } \\
\text { úlceras por pressão em adultos e idosos. } \\
\text { Pesquisa descritivo-exploratória e de abordagem } \\
\text { quantitativa. }\end{array}$ & $\begin{array}{l}\text { Pesquisa descritivo-exploratória e de abordagem } \\
\text { quantitativa. Foi utilizado questionário estruturado, a } \\
\text { partir do teste de conhecimento de Pieper. }\end{array}$ \\
\hline
\end{tabular}

REAS/EJCH | Vol.Sup.n.43 | e2553 | DOI: https://doi.org/10.25248/reas.e2553.2020 Página $\mathbf{5}$ de $\mathbf{1 3}$ 


\begin{tabular}{|c|c|c|c|}
\hline $\begin{array}{l}\text { 7-LIMA AFC et } \\
\text { al., (2015) }\end{array}$ & $\begin{array}{l}\text { Custos da implantação de um } \\
\text { protocolo de prevenção de } \\
\text { úlceras por pressão em um } \\
\text { hospital universitário. }\end{array}$ & $\begin{array}{l}\text { Calcular os custos diretos da implantação de um } \\
\text { protocolo de prevenção de LP em um hospital } \\
\text { universitário. }\end{array}$ & $\begin{array}{l}\text { Pesquisa quantitativa, exploratório-descritiva, do tipo } \\
\text { estudo de caso. Foram mapeadas as atividades } \\
\text { constituintes das etapas elaboração, implantação e } \\
\text { avaliação da implantação do protocolo. }\end{array}$ \\
\hline $\begin{array}{l}\text { 8-Silva CPS, et } \\
\text { al.(2016) }\end{array}$ & $\begin{array}{l}\text { Construção do Aplicativo para o } \\
\text { indicador de úlcera por pressão. }\end{array}$ & $\begin{array}{l}\text { Descrever as etapas da construção de um } \\
\text { software que auxilia na identificação dos fatores } \\
\text { de risco associados à úlcera por pressão. }\end{array}$ & $\begin{array}{l}\text { Descritivo sobre o desenvolvimento de um software } \\
\text { denominado: Aplicativo para o indicador de úlcera por } \\
\text { pressão. }\end{array}$ \\
\hline $\begin{array}{l}\text { 9-Moro JV e Caliri } \\
\text { MHL (2016) }\end{array}$ & $\begin{array}{l}\text { Úlcera por pressão após a alta } \\
\text { hospitalar e o cuidado em } \\
\text { domicílio. }\end{array}$ & $\begin{array}{l}\text { Identificar o perfil sociodemográfico e de saúde } \\
\text { de pacientes que necessitavam de cuidados } \\
\text { domiciliares após a alta, o nível de risco para } \\
\text { úlcera por pressão por meio da Escala de } \\
\text { Braden, e a prevalência de úlcera e o contexto do } \\
\text { cuidado domiciliar. }\end{array}$ & $\begin{array}{l}\text { Estudo transversal, com abordagem quantitativa, } \\
\text { aprovado pelo Comitê de Ética em Pesquisa da Escola } \\
\text { de Enfermagem de Ribeirão Preto. A coleta de dados } \\
\text { foi realizada nos domicílios por meio de entrevista e } \\
\text { inspeção da pele. }\end{array}$ \\
\hline $\begin{array}{l}\text { 10-Olkoski E e } \\
\text { Assis GM (2016) }\end{array}$ & $\begin{array}{l}\text { Aplicação de medidas de } \\
\text { prevenção para úlceras por } \\
\text { pressão pela equipe de } \\
\text { enfermagem antes e após uma } \\
\text { campanha educativa. }\end{array}$ & $\begin{array}{l}\text { Avaliar a efetividade de uma campanha para } \\
\text { prevenção de úlcera por pressão em um hospital } \\
\text { de ensino de Curitiba. }\end{array}$ & $\begin{array}{l}\text { Pesquisa exploratório-descritiva com abordagem } \\
\text { quantitativa. Consistiu na avaliação de medidas } \\
\text { preventivas aplicadas aos pacientes dos centros de } \\
\text { terapia intensiva e semi-intensiva, por meio de } \\
\text { avaliação leito a leito com checklist contendo as } \\
\text { medidas recomendadas. }\end{array}$ \\
\hline $\begin{array}{l}\text { 11-Toffoletto MC } \\
\text { et al. (2016) }\end{array}$ & $\begin{array}{l}\text { Fatores relacionados à } \\
\text { ocorrência de eventos adversos } \\
\text { em pacientes idosos críticos. }\end{array}$ & $\begin{array}{l}\text { Identificar os fatores relacionados à ocorrência } \\
\text { de eventos adversos em pacientes idosos } \\
\text { críticos internados em Unidade de Terapia } \\
\text { Intensiva segundo características demográficas } \\
\text { e clínicas. }\end{array}$ & $\begin{array}{l}\text { Estudo de coorte retrospectivo realizado em nove } \\
\text { unidades de um hospital universitário. Os dados foram } \\
\text { coletados dos prontuários e do acompanhamento de } \\
\text { passagens de plantão de enfermagem. }\end{array}$ \\
\hline
\end{tabular}

REAS/EJCH | Vol.Sup.n.43 | e2553 | DOI: https://doi.org/10.25248/reas.e2553.2020 Página 6 de 13 


\begin{tabular}{|c|c|c|c|}
\hline $\begin{array}{l}\text { 12-Braquehais } \\
\text { AR e Dallarosa } \\
\text { FS (2016) }\end{array}$ & $\begin{array}{lrr}\text { Conhecimento } & \text { dos } \\
\text { enfermeiros acerca } & \text { da } \\
\text { prevenção de lesões } & \text { por } \\
\text { pressão em unidade } & \text { de } \\
\text { terapia intensiva. } & \end{array}$ & $\begin{array}{l}\text { Investigar o perfil e o conhecimento dos } \\
\text { enfermeiros acerca da prevenção de úlceras por } \\
\text { pressão. }\end{array}$ & $\begin{array}{l}\text { Estudo transversal, descritivo, desenvolvido em três } \\
\text { Unidades de Terapia Intensiva de um hospital público } \\
\text { do estado do Ceará-Brasil, com } 20 \text { enfermeiros. }\end{array}$ \\
\hline $\begin{array}{l}\text { 13-Rios BL, et } \\
\quad \text { al.(2016) }\end{array}$ & $\begin{array}{l}\text { Prevenção de úlceras por } \\
\text { pressão em unidade de terapia } \\
\text { intensiva: Um relato de } \\
\text { experiência. }\end{array}$ & $\begin{array}{l}\text { Apresentar a experiência de uma atividade } \\
\text { educativa desenvolvida por estudantes do } 9 \text { o } \\
\text { semestre de Enfermagem da Universidade } \\
\text { Federal do Recôncavo da Bahia, durante o } \\
\text { período de estágio em uma unidade de terapia } \\
\text { intensiva. }\end{array}$ & $\begin{array}{l}\text { Estudo descritivo, do tipo relato de experiência, } \\
\text { referente a um projeto de intervenção do componente } \\
\text { curricular Estágio Supervisionado II, a partir do qual se } \\
\text { desenvolveu uma atividade educativa. Pesquisa } \\
\text { Convergente assistencial, realizada num distrito } \\
\text { sanitário do Sul do Brasil, com } 20 \text { enfermeiros. }\end{array}$ \\
\hline $\begin{array}{l}\text { 14-Medeiros LNB, } \\
\text { et al. (2017) }\end{array}$ & $\begin{array}{l}\text { Prevalência de úlceras por } \\
\text { pressão em unidades de terapia } \\
\text { Intensiva. }\end{array}$ & $\begin{array}{l}\text { Detectar a prevalência de lesão por Pressão (LP) } \\
\text { em pacientes internados em Unidades de } \\
\text { Terapia Intensiva (UTIs). }\end{array}$ & $\begin{array}{l}\text { Estudo transversal, de abordagem quantitativa, } \\
\text { desenvolvido em um hospital de referência para o } \\
\text { estado do Rio Grande do Norte em urgência e trauma, } \\
\text { situado no distrito sanitário leste do município de Natal } \\
\text { (RN), Brasil. }\end{array}$ \\
\hline $\begin{array}{l}\text { 15-Pereira AFM, } \\
\text { et al.(2017) }\end{array}$ & $\begin{array}{l}\text { Incidência de lesão por pressão } \\
\text { em um hospital universitário. }\end{array}$ & $\begin{array}{l}\text { Identificar a incidência de lesão por pressão nas } \\
\text { unidades de internação de um hospital } \\
\text { universitário da região nordeste do Brasil. }\end{array}$ & $\begin{array}{l}\text { Estudo quantitativo, prospectivo e descritivo, realizado } \\
\text { com } 114 \text { pacientes dos três postos de internação de um } \\
\text { hospital universitário da região nordeste do brasil. a } \\
\text { coleta de dados aconteceu durante } 60 \text { dias } \\
\text { consecutivos no período de } 03 \text { de agosto a } 01 \text { de } \\
\text { outubro de } 2015 \text {. }\end{array}$ \\
\hline $\begin{array}{l}\text { 16-Petz FFC, et } \\
\text { al.(2017) }\end{array}$ & $\begin{array}{l}\text { Úlcera por pressão em unidade } \\
\text { de terapia intensiva: estudo } \\
\text { epidemiológico. }\end{array}$ & $\begin{array}{l}\text { Analisar o perfil clínico dos pacientes portadores } \\
\text { e não portadores de úlcera por pressão. }\end{array}$ & $\begin{array}{l}\text { Estudo prospectivo, longitudinal, de abordagem } \\
\text { quantitativa, desenvolvido em um em uma Unidade de } \\
\text { Terapia Intensiva de um hospital privado de Curitiba } \\
\text { (PR), Brasil. }\end{array}$ \\
\hline $\begin{array}{l}\text { 17-Angelo CS et } \\
\text { al.(2017) }\end{array}$ & $\begin{array}{l}\text { Efetividade do protocolo } \\
\text { prevenção de lesões de pele em } \\
\text { cirurgias urológicas robóticas. }\end{array}$ & $\begin{array}{l}\text { Verificar a efetividade do Protocolo Prevenção de } \\
\text { Lesão de Pele, por meio do levantamento de } \\
\text { ocorrências causadas pelo posicionamento } \\
\text { cirúrgico em pacientes oncológicos submetidos } \\
\text { às cirurgias urológicas robóticas. }\end{array}$ & $\begin{array}{l}\text { Trata-se de uma pesquisa descritiva, retrospectiva, } \\
\text { abordagem quantitativa, referente ao ano } \\
\text { de } 2015 \text {. O estudo foi feito no centro cirúrgico de um } \\
\text { hospital oncológico que realiza em média } 1.000 \\
\text { cirurgias/mês }\end{array}$ \\
\hline
\end{tabular}

REAS/EJCH | Vol.Sup.n.43 | e2553 | DOI: https://doi.org/10.25248/reas.e2553.2020 Página 7 de 13 


\begin{tabular}{|c|c|c|c|}
\hline $\begin{array}{l}\text { 18-Vasconcelos } \\
\text { JMB e Caliri MHL } \\
\text { (2017) }\end{array}$ & $\begin{array}{l}\text { Ações de enfermagem antes e } \\
\text { após um protocolo de prevenção } \\
\text { de lesões por pressão em terapia } \\
\text { intensiva. }\end{array}$ & $\begin{array}{l}\text { Avaliar as ações dos profissionais de } \\
\text { enfermagem, antes e após utilização de } \\
\text { protocolo de prevenção de lesões por pressão, } \\
\text { em Unidade de Terapia Intensiva. }\end{array}$ & $\begin{array}{l}\text { Estudo observacional, prospectivo, comparativo, do } \\
\text { tipo antes e depois, com abordagem quantitativa, } \\
\text { realizado em hospital de ensino, na Paraíba. Foram } \\
\text { observadas as ações de enfermagem durante } 38 \\
\text { banhos no leito antes e } 44 \text { depois do protocolo. }\end{array}$ \\
\hline $\begin{array}{l}\text { 19-Debon } R \text {, et } \\
\text { al.(2018) }\end{array}$ & $\begin{array}{l}\text { A Visão de Enfermeiros Quanto } \\
\text { a Aplicação da Escala de Braden } \\
\text { no Paciente Idoso. }\end{array}$ & $\begin{array}{l}\text { Identificar se os enfermeiros têm conhecimento e } \\
\text { fazem uso correto em seu cuidado diário da } \\
\text { escala de Braden em idosos, instrumento } \\
\text { consolidado na prevenção das Lesões por } \\
\text { Pressão }\end{array}$ & $\begin{array}{l}\text { Pesquisa qualitativa, exploratória, descritiva, realizada } \\
\text { em um hospital ao Norte do RS. Os dados foram } \\
\text { coletados por meio de entrevistas semiestruturadas } \\
\text { com quatorze enfermeiros e analisados mediante } \\
\text { análise temática. }\end{array}$ \\
\hline $\begin{array}{l}\text { 20-Aroldi JBC, et } \\
\text { al.(2018) }\end{array}$ & $\begin{array}{l}\text { Percepção do impacto no } \\
\text { trabalho de um treinamento } \\
\text { online sobre prevenção de lesão } \\
\text { por pressão. }\end{array}$ & $\begin{array}{l}\text { Avaliar a percepção do impacto em amplitude e } \\
\text { o suporte à transferência do treinamento on-line } \\
\text { sobre prevenção de lesão por pressão. }\end{array}$ & $\begin{array}{l}\text { Estudo exploratório-descritivo, desenvolvido no } \\
\text { município de São Paulo, no Hospital Universitário da } \\
\text { Universidade de São Paulo. A população da pesquisa } \\
\text { abrangeu } 97 \text { enfermeiros que atuavam em unidades } \\
\text { com pacientes de maior risco de desenvolvimento de } \\
\text { lesão por pressão. }\end{array}$ \\
\hline $\begin{array}{l}\text { 21-Soares CF e } \\
\text { Heidemann ITSB } \\
\qquad(2018)\end{array}$ & $\begin{array}{l}\text { Promoção da saúde e prevenção } \\
\text { da lesão por pressão: } \\
\text { expectativas do enfermeiro da } \\
\text { atenção primária. }\end{array}$ & $\begin{array}{l}\text { Apresentar a aplicabilidade da Escala de Braden } \\
\text { na percepção dos enfermeiros da atenção } \\
\text { primária, e identificar as medidas de prevenção, } \\
\text { e promoção da saúde de modo evitar o } \\
\text { desenvolvimento da lesão por pressão. }\end{array}$ & $\begin{array}{l}\text { Pesquisa Convergente assistencial, realizada num } \\
\text { distrito sanitário do Sul do Brasil, com } 20 \text { enfermeiros. } \\
\text { A coleta de dados ocorreu em julho de } 2014 \text {, através } \\
\text { de entrevista e de uma prática educativa na forma de } \\
\text { oficina temática intitulada "Diga não à lesão por } \\
\text { pressão, prevenir é o melhor cuidado". }\end{array}$ \\
\hline
\end{tabular}

Fonte: Feitosa DVS, et al., 2019. 


\section{DISCUSSÃO}

Após análise e da leitura de artigos, evidenciou-se a importância da discussão das temáticas: Escala de Braden e medidas preventivas relacionadas às boas práticas assistenciais.

\section{Escala de Braden}

No contexto atual, em que a segurança do paciente, a humanização e qualidade dos cuidados têm sido amplamente discutidos e valorizados, e a incidência alta de LP é apontada como um indicador negativo da qualidade. A avaliação e a prescrição de cuidados com a pele são uma atribuição do enfermeiro, assim, é fundamental que o enfermeiro utilize conhecimentos atualizados e use estratégias para planejar a assistência de modo a prevenir as LP e proporcionar a qualidade da assistência (NOGUEIRA PC, et al., 2015).

As escalas preditivas da lesão por pressão têm se configurado em um importante instrumento no reconhecimento dos pacientes que apresentam risco de desenvolvê-la. Entre os vários métodos de avaliação para o risco de LP, a Escala de Braden é a mais difundida e utilizada, por ter sido submetida a diversos estudos e testes de confiabilidade e validade em diferentes populações (BRAQUEHAIS AR e DALLAROSA FS, 2014).

A Escala de Braden é um instrumento útil, de fácil uso, não tendo custo para a instituição e usado como um indicador de qualidade da saúde na segurança do paciente como instrumento preventivo, uma vez que auxilia o enfermeiro para a realização de uma avaliação global do risco de formação de LP no paciente hospitalizado, para que assim possa se tomar os cuidados necessários com o objetivo de eliminar o risco (DEBON R, et al., 2018).

A referida escala é composta por seis subdivisões que analisam o grau de percepção sensorial, umidade, atividade física, nutrição, mobilidade, fricção e cisalhamento. Essas subdivisões existentes na escala de Braden são graduadas de 1 a 4, com exceção da parte referente a fricção e cisalhamento, que varia é de 1 a 3. Nesse sentido, o escore total varia de 6 a 23, tendo como classificação os escores de 19 a 23 que indicam pacientes sem risco, de 15 a 18 baixo risco, de 13 a 14 risco moderado, de 10 a 12 alto risco e igual ou menor que 9, altíssimo risco (BRAQUEHAIS AR e DALLAROSA FS, 2014).

Ainda, nesse sentido, a escala de Braden, avalia e contabiliza os fatores que contribuem à redução da tolerância tecidual à compressão prolongada, por meio de seis subescalas: percepção sensorial, umidade, atividade, mobilidade, nutrição, fricção e cisalhamento. Nesse contexto, o enfermeiro possui competência para identificar os fatores de risco para o desenvolvimento da lesão por pressão a partir da avaliação dos mesmos, sendo imprescindível que esta classificação seja adequada, para favorecer a melhoria da assistência de enfermagem através do estabelecimento de protocolos de prevenção segundo o nível de risco do paciente (PEREIRA AFM, et al., 2017).

Assim, a utilização de escalas, como a Escala de Braden de avaliação de risco para lesão por pressão, requer pouca habilidade do profissional enfermeiro, porém demanda mais tempo da assistência para sua avaliação, como também é necessário o envolvimento da equipe para que se concretize o trabalho, uma vez que o enfermeiro avalia o risco, porém os técnicos de enfermagem devem colocar em prática o cuidado prescrito, no atendimento diário (DEBON R, et al., 2018).

A utilização da Escala Braden proporciona qualidade da assistência ao paciente com risco de lesão por pressão, já que possibilita o conhecimento do perfil do paciente e direciona a sistematização do cuidado. Ademais, é de suma importância a implementação da Sistematização da Assistência de Enfermagem (SAE) que faz o levantamento de questionamentos para o risco e elaboração de planos de cuidados de forma individualizada (ROCHA LES, et al., 2015). Nessa perspectiva, o enfermeiro, enquanto profissional de saúde, envolvido na assistência do paciente, deve planejar as intervenções de enfermagem capaz de promover a saúde, a adesão ao tratamento e estimular o autocuidado. Essas práticas devem também levar em conta as crenças, valores e aspectos culturais que influenciam diretamente na evolução do paciente, relacionados aos componentes cognitivo, afetivo e comportamental, como também ao aprendizado, à decisão de se cuidar e ao cuidado de si mesmo (MORO JV e CALIRI MHL, 2016). 
Na prevenção da LP é imprescindível os ajustes nutricionais e as intervenções para auxiliar a mobilização ou mobilidade dos pacientes, entre outras medidas, sendo assim é importante a participação da equipe multiprofissional. Sob tal perspectiva, a Escala de Braden é indispensável para avaliar o risco de surgimento da LP, porém é necessário que toda a equipe compreenda os critérios de classificação (DEBON R, et al., 2018).

A vista disso cresce a responsabilidade da prevenção das lesões por parte de toda a equipe multiprofissional, uma vez que os trabalhadores da saúde devem ser compromissados e intervir como instrumento de melhoria das condições de vida e saúde dos pacientes (RIOS, et al., 2016). Diante da magnitude das repercussões causadas pela lesão por pressão, faz-se necessária atualização contínua da equipe multiprofissional, principalmente da equipe de enfermagem (SOARES CF e HEIDEMANN ITSB, 2018).

\section{Medidas preventivas relacionadas às boas práticas assistenciais}

Os cuidados associados à prevenção e às condutas de promoção a saúde surgiram de elementos comuns adotados pelos profissionais, os quais foram nomeados e apresentados no Quadro 2. Neste contexto, que discute as ideias direcionadas a prevenir a lesão por pressão e aumentar a qualidade de vida dos pacientes suscetíveis ao acontecimento desses agravos, foram mencionadas medidas de prevenção com a pele, que contemplam a inspeção, higienização, hidratação e a sua proteção.

Para Laurenti TC, et al. (2015), o aparecimento das lesões por pressão em consequência está atrelado a não utilização de ações preventivas, a escassez de recursos materiais apropriados e importantes para cada quadro, além da pela falta de registros de enfermagem sendo determinado pela ausência da checagem da enfermagem ou ainda preenchimento inapropriado do documento.

A existência de lesão por pressão repercute em circunstâncias estressantes para os profissionais da saúde, principalmente para a equipe de enfermagem, que lida com curativos e medidas preventivas para preservar a integridade da pele dos pacientes. Toda essa responsabilidade, junto com outras atividades, como administração de medicamentos e banho no leito, ocasiona um excesso de trabalho para a equipe e pode ser um fator para contribuir para a formação das lesões, visto que a qualidade da assistência fica comprometida (MEDEIROS LNB, et al., 2017). Além disso, As LPs afetam a saúde do paciente nos aspectos físicos, psíquicos e emocionais, além disso, preocupam os familiares quanto às consequências que essas lesões podem trazer ao indivíduo acometido, como também elevam o custo financeiro para a instituição, devido ao investimento em produtos para o tratamento (MEDEIROS LNB, et al., 2017).

Nesse sentido, a manutenção da integridade da pele deve ser uma das finalidades da equipe de enfermagem e essa assistência deve ser norteada em evidências científicas. Embora se reconheça a multicausalidade da LPs e a necessidade de uma abordagem multidisciplinar, a equipe de enfermagem é responsável pela assistência direta e contínua aos pacientes, o que the confere o principal destaque na prevenção desse problema (VASCONCELOS JMB e CALIRI MHL, 2017). Sob tal perspectiva, as estratégias de prevenção são um dos métodos de maior eficácia disponível para minimizar a ocorrência de lesão por pressão em instituições de internação hospitalar e a gestão de riscos, sendo essencial a sua implementação e avaliação de medidas preventivas. Dessa forma, devem ser realizados treinamentos contínuos com a equipe, para capacitar os profissionais a identificar potenciais fatores de riscos e a tomar medidas de preventivas (LAURENTI TC, et al., 2015).

As LP podem ser prevenidas através de medidas adequadas, educação dirigida aos pacientes e seu cuidador, com estratégias que viabilizem à continuidade do cuidado no domicílio. Para que as mesmas possam ser prevenidas, é essencial conhecer os fatores de risco que os pacientes apresentam para 0 aparecimento dessas lesões. Cabe ao enfermeiro o planejamento, a execução e a avaliação de intervenções educativas para familiares quanto às medidas preventivas (NOGUEIRA PC, et al., 2015). Portanto, o conhecimento das medidas de prevenção e das características da lesão por pressão deve fazer parte do conhecimento de todos os profissionais da área de enfermagem (ROCHA LES, et al., 2015). Para promover a segurança do paciente, como também, reduzir a exposição aos eventos adversos, Laurenti TC, et al. (2015) afirmam que é essencial reforçar o acompanhamento e preenchimento dos protocolos de gerenciamento de riscos para controle e prevenção. 
Nesse sentido, a aplicação da Escala de Braden pelo profissional enfermeiro direciona as intervenções de enfermagem na prevenção de Lesão por Pressão. Estudos científicos realizados por Barbosa TP, et al. (2014), ao associar as medidas de prevenção, foi observado que a assistências prestada não foram realizadas com base na classificação da Escala de Braden, ou seja, as prescrições das intervenções de enfermagem não estavam relacionadas com o risco que o paciente estava exposto.

Observou-se também que, devem ser efetivadas, realizadas medidas que reduzam a umidade e controlem ○ PH. O aporte nutricional balanceado também foi mencionado em algumas literaturas, assim como o reposicionamento planejado em angulação e com maior frequência (Quadro 2).

Quadro 2 - Medidas preventivas para lesão por pressão. Aracaju - SE, 2019.

\begin{tabular}{|c|c|}
\hline Autor/ano & Principais resultados \\
\hline $\begin{array}{l}\text { Soares CF e } \\
\text { Heidemann } \\
\text { ITSB (2018) }\end{array}$ & $\begin{array}{l}\text { Examinar diariamente a pele de maneira detalhada através da inspeção e palpação; } \\
\text { Oportunizar o exame da pele durante o banho; Atentar para a temperatura da água durante } \\
\text { o banho; Manter a pele livre dos fluidos corporais, fazendo limpeza com algodão úmido com } \\
\text { água; Utilizar na pele hidratante e emoliente; Oferecer adequada ingesta hídrica e aporte } \\
\text { nutricional equilibrado; Fazer uso de placas de proteção da pele; Evitar aplicar na pele } \\
\text { produtos com elevado teor alcoólico. }\end{array}$ \\
\hline $\begin{array}{l}\text { Laurenti TC, } \\
\text { et al. }(2015)\end{array}$ & $\begin{array}{l}\text { Realizar mudança de decúbito de } 2 / 2 \mathrm{~h} \text {; Cabeceira elevada em até } 30^{\circ} \text {; Lençóis de cama } \\
\text { secos e limpos; Colchão redutor de pressão; Não massagear áreas com hiperemia; Usar } \\
\text { forro móvel para mover paciente acamado; Estimular deambulação; Realizar exercícios } \\
\text { ativos e/ou passivos no paciente; Realizar higiene íntima somente com água e sabão neutro; } \\
\text { Aplicar protetor cutâneo; Manter paciente em decúbito lateral inclinado } 30^{\circ} \text { em relação à } \\
\text { cama; Manter protetores entre as proeminências ósseas em contato; Mobilizar paciente no } \\
\text { leito sem arrastar; Observar integridade cutânea e mucosa; Proteger proeminências ósseas; } \\
\text { Colocar uripen ou fralda na presença de incontinência. }\end{array}$ \\
\hline $\begin{array}{l}\text { Inque KC e } \\
\text { Matsuda LM } \\
\text { (2015) }\end{array}$ & Filme transparente de poliuretano e a placa hidrocoloide. \\
\hline $\begin{array}{l}\text { Barbosa TP, } \\
\text { et al. }(2014)\end{array}$ & $\begin{array}{l}\text { A hidratação da pele do paciente, Camas limpas; Pacientes limpos e secos; Mudança de } \\
\text { decúbito de } 2 \text { em } 2 \text { horas; Colchões piramidais; Coxins. }\end{array}$ \\
\hline $\begin{array}{ll}\text { Olkoski } & \text { E e } \\
\text { Assis } & \text { GM } \\
(2016) & \end{array}$ & $\begin{array}{l}\text { Mudança de decúbito; hidratação da pele com óleo; colchão caixa de ovo; uso de coxins e } \\
\text { massagem. Outro estudo demonstrou o uso de luvas com água e a hidratação com óleo de } \\
\text { girassol. }\end{array}$ \\
\hline $\begin{array}{l}\text { Maurício AB, } \\
\text { et al. }(2014)\end{array}$ & $\begin{array}{l}\text { Planejar ações de prevenção por meio da Promoção de intervenções educativas que tragam } \\
\text { com clareza as respostas para as dúvidas da equipe de enfermagem com relação à } \\
\text { prevenção da LP. }\end{array}$ \\
\hline $\begin{array}{l}\text { Lima AFC, et } \\
\text { al. }(2015)\end{array}$ & $\begin{array}{l}\text { A elaboração e implementação de protocolos assistenciais afim de possibilitar melhora na } \\
\text { qualidade da assistência, reduzindo as complicações decorrentes da ocorrência de LP. }\end{array}$ \\
\hline $\begin{array}{l}\text { Toffoletto } \\
\text { MC, et al. } \\
(2016)\end{array}$ & Diminuição do tempo de internação, inspeção diária da pele e mudança de decúbito. \\
\hline $\begin{array}{l}\text { Petz FFC, et } \\
\text { al. }(2017)\end{array}$ & Avaliação do risco para lesão por pressão por meio da escala de Braden. \\
\hline $\begin{array}{l}\text { Angelo CS, et } \\
\text { al. (2015) }\end{array}$ & Posicionamento adequado durante o procedimento cirúrgico e utilização de coxins. \\
\hline
\end{tabular}

Fonte: Feitosa DVS, et al., 2019. 
Como medidas de prevenção Soares CF e Heidemann ITSB (2018), retratam que o cuidado voltado ao ambiente também se faz necessário, como lençóis de camas limpos e esticados, equipamentos de redistribuição da pressão (coxins, travesseiros), o uso colchão apropriados e a manutenção adequada deste. Já no que diz respeito à promoção de saúde, dados revelam que a participação do paciente no autocuidado, assim como dos seus familiares, cuidadores e sociedade, como responsáveis e, acima de tudo, personagens importantes no processo de viver saudável é essencial para evolução do seu quadro.

Em um estudo realizado por Inoue KC e Matsuda LM (2015) apesar da placa de hidrocoloide apresentarse mais efetiva para o desfecho intermediário da LP, os resultados recomendam que a utilização de filme transparente de poliuretano apresenta mais benefícios para a prevenção de lesão por pressão em região sacra. O filme transparente comprova ser mais custo-efetivo do que o hidrocoloide como medida preventiva de LP em região sacral entre pacientes de terapia intensiva e com valores menos custosos que o hidrocoloide.

A hidratação da pele do paciente também é uma medida de prevenção a ser utilizada com a finalidade de reduzir o risco de aparecimento de LP, tendo em vista que os pacientes sujeitos à hidratação mostram escores superiores em comparação aqueles que não tiveram (BARBOSA TP, et al., 2014).

No que se refere ao ângulo de lateralização e elevação de cabeceira, autores declaram que deverá ser utilizado $30^{\circ}$ em posição semi-Fowler se o paciente suportar estas posições, alternando entre o lado esquerdo, dorsal e lado direito (OLKOSKI e ASSIS GM, 2016).

Diante do exposto, o uso apropriado das intervenções selecionadas trará um menor custo emocional e financeiro para o paciente, para o familiar e para o Sistema de Saúde. Ademais, torna-se necessário ampliar e estimular o planejamento e implementação de ações que proporcionem o acesso à capacitação profissional, com o objetivo de ampliar e melhorar a assistência ofertada aos pacientes (BRAQUEHAIS AR e DALLAROSA FS, 2014).

\section{CONSIDERAÇÕES FINAIS}

A lesão por pressão tornou-se um problema de saúde pública devido a sua alta incidência, característica crônica e recorrente, que ocasiona tratamentos longos e complexos. Quanto às medidas preventivas, foram mencionadas ações de prevenção que contemplam a inspeção, higienização e hidratação. Assim, torna-se relevante a atuação da equipe multiprofissional, especialmente a equipe de enfermagem, a adoção de medidas voltadas para prevenção e condução deste agravo na instituição, sendo essencial associar teoria e prática baseadas em diretrizes com evidências que possam conduzir à prática clínica efetiva, com o intuito de se minimizar a incidência de LP. Por fim, ressalta-se a importância desse estudo para ampliar a conhecimento acerca da assistência, especialmente a elaboração e execução de medidas de prevenção da lesão por pressão.

\section{REFERÊNCIAS}

1. ANGELO CS, et al. Efetividade do protocolo prevenção de lesões de pele em cirurgias urológicas robóticas. REV. SOBECC, 2017, 22(3), 152-160.

2. AROLDI JBC, et al. Percepção do impacto no trabalho de um treinamento online sobre prevenção de lesão por pressão. Texto Contexto de Enfermagem, 2018, 27(3),1-12.

3. BARBOSA TP, et al. Avaliação do risco de úlcera por pressão em UTI e assistência preventiva de enfermagem. Revista de Enfermagem UERJ, 2014, 22(3),353-358.

4. BRAQUEHAIS AR, DALLAROSA FS. Conhecimento dos enfermeiros acerca da prevenção de lesões por pressão em unidade de terapia intensiva. Revista de Enfermagem UFPI, 2016, 5(4), 13-18.

5. DEBON R, et al. A Visão de Enfermeiros quanto a aplicação da Escala de Braden no paciente idoso. Revista de Pesquisa: Cuidado é Fundamental Online,2018, 10(3),817-823.

6. INOUE KC, MATSUDA LM. Avaliação de Custo-Efetividade de Dois Tipos de Curativos Para Prevenção de Úlcera Por Pressão. Acta Paulista de Enfermagem, 2015, 28(5), 415-419.

7. LAURENTI TC et al. Gestão Informatizada de Indicadores de Úlcera Por Pressão. Journal Health Informatics, 2015 , 7(3), 94-98. 
8. LIMA AFC, et al. Custos da implantação de um protocolo de prevenção de úlceras por pressão em um hospital universitário. Rev. Eletr. Enf. [Internet], 2015, 17(4).

9. MAURICIO AB, et al. Conhecimentos dos profissionais de enfermagem sobre úlceras por Pressão na Clínica Médica. Rev Enferm UFPI, 2014, 3(3), 5-11.

10. MORO JV, CALIRI MHL. Úlcera por pressão após a alta hospitalar e o cuidado em domicílio. Escola Anna Nery, 2016, 20(3),1-6.

11. MEDEIROS LNB, et al. Prevalência de úlceras por pressão em unidades de terapia intensiva. Revista de Enfermagem UFPE, 2017,11(7),697-703.

12. NPUAP. National Pressure Ulcer Advisory Panel. European Pressure Ulcer Advisory Panel (EPUAP); Pan Pacific Injury Alliance (PPPIA). Prevention and treatment of pressure ulcers. Osborn Park, Western Australia. Clinical Practice Guideline, 2014.

13. NOGUEIRA PC, et al. Conhecimento dos cuidadores de indivíduos com lesão medular sobre prevenção de úlcera por pressão. Aquichan, 2015, 15(2),188-199.

14. OLKOSKI E, ASSIS GM. Aplicação de medidas de prevenção para úlceras por pressão pela equipe de enfermagem antes e após uma campanha educativa. Escola Anna Nery, 2016, 20(2), 363-369.

15. PEREIRA AFM, et al. Incidência de lesão por pressão em um hospital universitário. Revista de Enfermagem UFPI, 2017,6(1),36-39.

16. PETZ FFC, et al. Úlcera por pressão em unidade de terapia intensiva: estudo epidemiológico. Rev enferm UFPE on line, 2017, 11(1), 287-95.

17. ROCHA LES, et al. Prevenção de úlceras por pressão: Avaliação do conhecimento dos profissionais de enfermagem. Cogitare Enfermagem, 2015, 20(3), 509-604.

18. RIOS BL, et al. Prevenção de úlceras por pressão em unidade de terapia intensiva: um relato de experiência. Revista de Enfermagem UFPE, 2016,10(6),4959-4964.

19. SILVA CPC, et al. Construção do Aplicativo para o indicador de úlcera por pressão. Journal Health Informatics, 2016, 8(4),134-141.

20. SOARES CF, HEIDEMANN ITSB. Promoção da saúde e prevenção da lesão por pressão: expectativas do enfermeiro da atenção primária. Texto Contexto de Enfermagem, 2018, 27(2), 2018.

21. TOFFOLETTO MC, et al. Fatores relacionados à ocorrência de eventos adversos em pacientes idosos críticos. Rev Bras Enferm [Internet], 2016, 69(6), 1039-45.

22. VASCONCELOS JMB, CALIRI MHL. Ações de enfermagem antes e após um protocolo de prevenção de lesões por pressão em terapia intensiva. Escola Anna Nery, 2017, 21(1), 1-10. 